\title{
“LYING” IN THE PIGEON
}

\author{
Robert P. Lanza, James Starr, and B. F. Skinner \\ UNIVERSITY OF PENNSYLVANIA AND HARVARD UNIVERSITY
}

\begin{abstract}
Two pigeons were taught to use symbols to communicate information about hidden colors to each other. When reporting red was more generously reinforced than reporting yellow or green, both birds passed through a period in which they "lied" by reporting another color as red.
\end{abstract}

Key words: communication, symbolic matching, colors, "lying," pigeons

A sustained conversation in which one pigeon communicated information to another through the use of symbols has been described by Epstein, Lanza, and Skinner (1980). The pigeons pecked labeled keys on adjoining keyboards (Figure 1). The pigeon on the left (called "Jack") could obtain food only by correctly selecting the color on his keyboard that corresponded to a hidden color accessible only to the other pigeon ("Jill"). Jack "asked for Jill's help" by pecking and illuminating a key marked WHAT COLOR? In response, Jill looked at the hidden color (either red, green, or yellow) and pecked the corresponding black and white symbol key. To assure continued help, Jack depressed the key marked THANK YOU, rewarding Jill with brief access to mixed grain. Jack then looked at the symbol and pecked the key bearing the corresponding color. Correct selections were automatically reinforced with food. Jack then requested the name of the next color (selected pseudo-randomly). Here we report the effect of a change in the contingencies that led to behavior that in the human species would be called "lying."

In a separate study the pigeons had been conditioned over a period of several weeks to play the roles of both speaker and listener (Epstein \& Skinner, 1981). The following modifications were then made. When the hidden color was red, a correct report was reinforced with access to mixed grain for the usual length

We thank Scott Randall for helpful comments. The work was supported by NSF grant BNS-8007342. Send reprint requests to B. F. Skinner, Department of Psychology and Social Relations, Harvard University, Cambridge, Massachusetts 02138. of time (approximately $3.8 \mathrm{sec}$ ). When the color was either green or yellow, the reinforcement was much shorter. Grain remained accessible only from the time the listener pecked THANK YOU until the corresponding color was struck. When the listener was Jack, food was accessible to Jill for an average of $.72 \mathrm{sec}$ for green and $1.3 \mathrm{sec}$ for yellow. When the listener was Jill, the times for Jack were .75 and $.74 \mathrm{sec}$, respectively. These conditions were maintained for two to three weeks in daily sessions of 75 complete exchanges.

The speakers' responses to the $R, Y$, and $G$ keys during the last 15 sessions prior to the change and the first 15 sessions following the change are shown in Figure 2. Before the change, indicated by the vertical dotted lines, the durations of reinforcement for all correct responses were the same. Shortly after the change, when " $R$ " was more generously reinforced, both pigeons correctly reported " $R$ " more often. Jill reached complete accuracy. When the hidden color was green, Jack soon called it red $60 \%$ of the time. He called yellow red even more often. Jill also "lied" (but only about yellow), in spite of the precision with which she identified red.

Throughout the experiments false responses were never reinforced and were followed by the darkening of the apparatus for several seconds. By the fifteenth day of biased reinforcement, the performances had essentially returned to normal.

In summary, when correctly reporting a red color was more strongly reinforced than reporting yellow or green, both pigeons passed through a period in which they reported another color as red. Was this done with "intent 


\section{Hidden color}

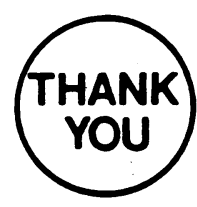

\section{WHAT COLOR?}

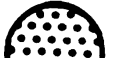

$\because \because 0$
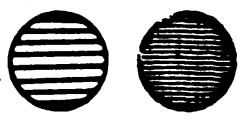

R

G

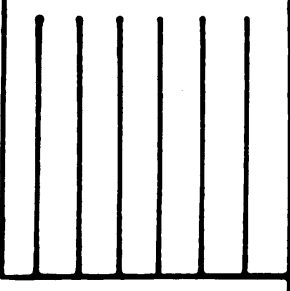

Listener's keyboard

\section{Speaker's keyboard}

Fig. 1. Adjoining keyboards for the pigeons. The listener (on the left) needs information about the color recessed $5 \mathrm{~cm}$ behind the curtain in the upper righthand corner of the speaker's keyboard on the right. The $R$, G, and $Y$ on the speaker's keyboard are black on white. The three keys below the WHAT COLOR? key on the listener's keyboard are yellow, red, and green from left to right.

to deceive"? If intention is only an indirect way of referring to the consequences responsible for the intentional behavior, then the answer would appear to be yes. The listener, given a wrong name, was deceived in the sense of then making an ineffective response. True, it was the experimenter who intercepted the reinforcement, but it is society not an individual who "punishes lying."

\section{REFERENCES}

Epstein, R., Lanza, R. P., \& Skinner, B. F. Symbolic communication between two pigeons (Columba livia domestica). Science, 1980, 207, 543-545.

Epstein, R., \& Skinner, B. F. The spontaneous use of memoranda by pigeons. Behaviour Analysis Letters, 1981, 1, 241-246.

Received May 18, 1982

Final acceptance May 19, 1982 


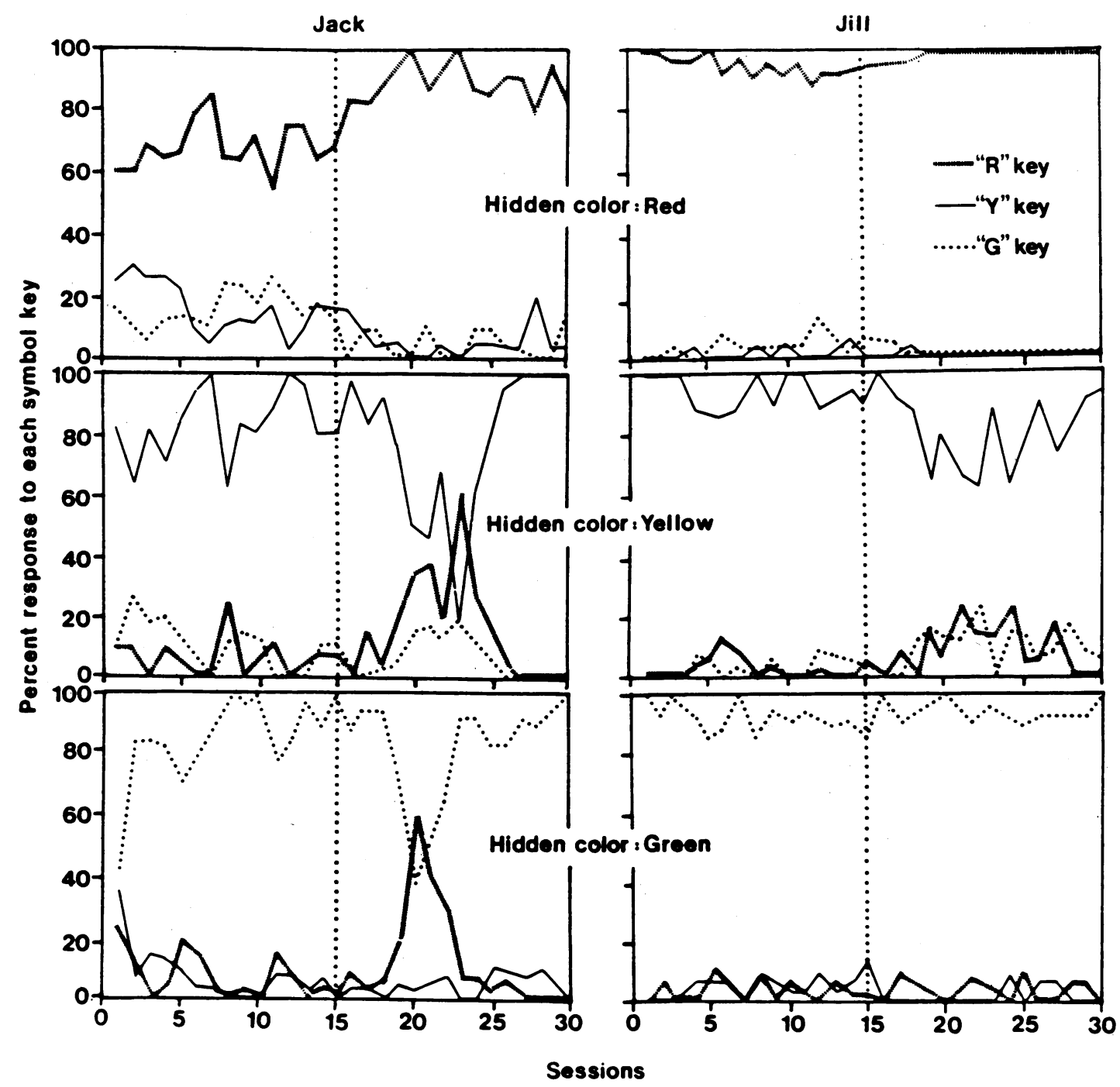

Fig. 2. "Lying" in the pigeons. The two columns show the speakers' responses to the $R$, Y, and G keys when the hidden color was red, yellow, or green. To the right of the vertical dotted lines reporting red was more generously reinforced than reporting yellow or green. 